\title{
Theoretical DFT Study of the Antioxidant Activity of Five Plant Food Benzoic Acid Derivatives: Dearomatization and Stability of H Abstraction Radicals
}

Bienfait Kabuyaya Isamura ${ }^{1,2, *}$, Issofa Patouossa ${ }^{3}$, Isaac Elaka Kaba ${ }^{4}$, Aristote Matondo ${ }^{2}$, and Pius Tshimankinda Mpiana ${ }^{2, *}$

${ }^{1}$ Department of Chemistry, Rhodes University, 6140, Makhanda, South Africa

${ }^{2}$ Department of Chemistry, Faculty of Sciences, University of Kinshasa, Kinshasa XI, Democratic Republic of the Congo

${ }^{3}$ Physical and Theoretical chemistry unit, Laboratory of applied physical and analytical chemistry, Faculty of Sciences, University of Yaoundé I, Yaoundé, Cameroon

${ }^{4}$ College of Pharmacy and Pharmaceutical Sciences, University of Toledo, Toledo, Ohio, USA Corresponding authors: isamurabft@gmail.com, ptmpiana@gmail.com 


\begin{abstract}
This study reports a computational investigation on the antioxidant activity of five plant food benzoic acid derivatives, namely gallic acid (GA), para-hydroxybenzoic acid (PHBA), protocatechuic acid (PCA), syringic acid (SA), and vanillic acid (VA). Based on computed thermodynamics parameters, a detailed comparative debate is developed concerning their free radical scavenging activity in the gas phase and polar solutions (in water and methanol solvents). This discussion goes on to elucidate both the most preferred mechanism and the order of antioxidant activity for these molecules in each environment. Paradoxically, calculations using the harmonic oscillator model of aromaticity (HOMA) suggest that $\mathrm{H}$ abstraction radicals gain in stability as the central benzene ring loses in structural aromaticity. Finally, spin densities and fukui function $\mathrm{f}^{0}$ seem to be good indicators of the local reactivity of these compounds towards free radicals.
\end{abstract}

Keywords: Antioxidant activity, Dearomatization, frontier molecular orbital, fukui function $\mathrm{f}^{0}$, DFT 


\section{Introduction}

The antioxidant activity is one of the most important properties of food plants [1]. An antioxidants is any substance occurring at lower concentrations to that of an oxidizable substrate, and capable of significantly decaying or even preventing the oxidation of that substrate [2]. The human body, in fact, produces powerful antioxidants such as alpha-lipoic acid and glutathione [3]. However, these natural "soldiers" may not be at the level of the expectations. Therefore, it is often recommended to regularly supply the body with external sources of antioxidants [4]. Antioxidants are naturally found in plants [5][6][7][8], animals [9][10], and microorganisms [11][12] or they may be synthesized by chemical means [13][14]. With the perpetual need to identify more effective antioxidant candidates to integrate into the preventive or therapeutic pipeline of several pathologies, the isolation and synthesis of new antioxidants is a hot research domain attracting the interest of a myriad of scientists [15][16][17][18].

Over the past decades, numerous studies have documented the antioxidant activity of benzoic acid derivatives (BADs). To cite only a few, Velika et al experimentally studied the antioxidant activity of fourteen benzoic acid derivatives against superoxide radical using the Beauchamp-Fridovick method [19]. Their study revealed that monohydroxybenzoic derivatives had the best antioxidant power. The same authors also noticed that molecules bearing the $-\mathrm{OH}$ group in ortho and para positions to the carboxylic group showed the highest activity. On the same track, Ali-Haidari et al isolated a series of benzoic acid derivatives from Cassia italica, a plant growing in South arabia, and showed them to display considerable antioxidant activities in DPPH assays, reaching up to $95.8 \%$ as compared to butylated hydroxyanisole $(\sim 94 \%)$ [20]. Furthermore, keeping in mind the need to identify potential natural substances that can reduce the severity of alcoholic liver disease (ALD), a disease known to be related with oxidative stress, several investigations have been conducted and many more are still popping up. In this vein, Saravanan and coworkers showed that 2-Hydroxy-4-methoxy benzoic acid (HMBA), the active principle of Hemidesmus indicus, significantly inhibits the development of liver injury in ethanol administration [21]. 
Beside experimental works, the literature also reports a panoply of theoretical studies that have contributed to unveiling the magic behind the antioxidant properties and site selectivity of many BADs. For instance, the homolytic and heterolytic splitting-off of the phenolic-OH groups of gallic acid and its carboxylic anion in gas-phase, benzene and water was elucidated by Skorna et al using the B3LYP functional combined with the $6-311++\mathrm{G}(\mathrm{d}, \mathrm{p})$ basis set [22]. These authors found that the deprotonation of the carboxylic groups results in an increase in the antioxidant activity in all environments. Another outstanding DFT study was conducted in gas phase to examine the effect of intramolecular hydrogen bonding and the presence of bulky fragments near the $-\mathrm{OH}$ group on the antioxidant activity of a series of vitamins and phenolic acids, including syringic and vanillic acids. The calculations performed suggested that intramolecular H-bonds involving ortho-hydroxy groups resulted in higher antioxidant power by lowering bond dissociation enthalpies and ionization potentials [23]. In 2012, Kalita et al excellently studied the antioxidant activity of gallic acid derivatives [24]. Their work brought unquestionable theoretical evidence that reactivity descriptors derived from conceptual density functional theory can be used to gain insights into the local reactivity of these antioxidants.

Furthermore, the literature indicates that phenolic antioxidants $\mathrm{ArO}-\mathrm{H}$ protect against oxidative stress according to three possible mechanisms. In the first model, known as Hydrogen Atom Transfer (HAT), ArO-H donates an $\mathrm{H}$ atom to a reactive free radical $\mathrm{R}^{\circ}$, which is then converted into a neutral species $\mathrm{RH}$ alongside a less reactive $\mathrm{ArO}^{\bullet}$ radical [23]. The second protocol is the Stepwise Electron Transfer Proton Transfer (SET-PT) pathway, where ArO-H consecutively withdraws one electron and a proton onto the free radical, leading to the same products as in the HAT route [25]. The third model [Sequential Proton Loss Electron Transfer (SPLET)] involves the heterolytic dissociation of an O-H bond, followed by the subsequent shift of a single electron from $\mathrm{ArO}^{-}$to the free radical [26]. Although the net result of these three mechanisms is the same, i.e. the formation of less harmful radicals, it has been shown that under certain conditions one of them always prevails [7]. Following the HAT mechanism, one can assess the antioxidant activity of a given molecule ArO-H by computing the bond dissociation enthalpies 
(BDE) of all the $\mathrm{O}-\mathrm{H}$ bonds in the molecule. This allows for the measurement of the amount of energy required to break a bond in a homolytic fashion, so that small BDEs can be related to higher antioxidant activity [5]. In the context of the SET-PT mechanism, the debate is grounded on two indicators, namely the ionization potential (IP) and proton dissociation enthalpy (PDE). Low IPs and PDEs are generally associated with great antioxidant properties [27]. Proton affinities (PA) and electron transfer enthalpies (ETE) are the most suitable thermodynamics parameters to discuss the antioxidant power with respect to the SPLET protocol. A rule of thumb is that the lower the PAs and ETEs are, the higher the antioxidant activity [28].

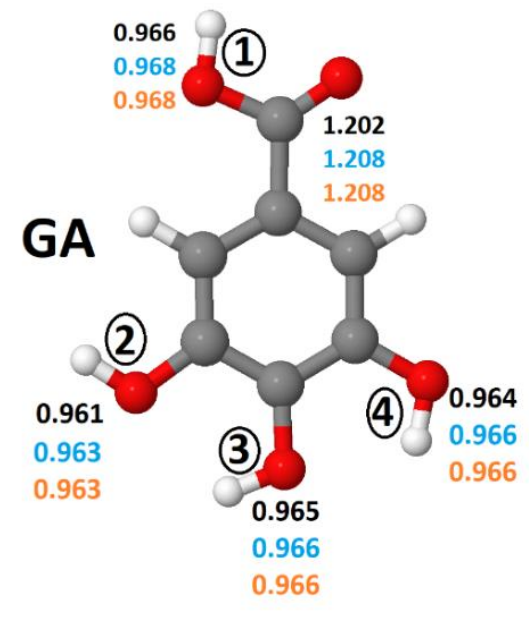

Gas phase

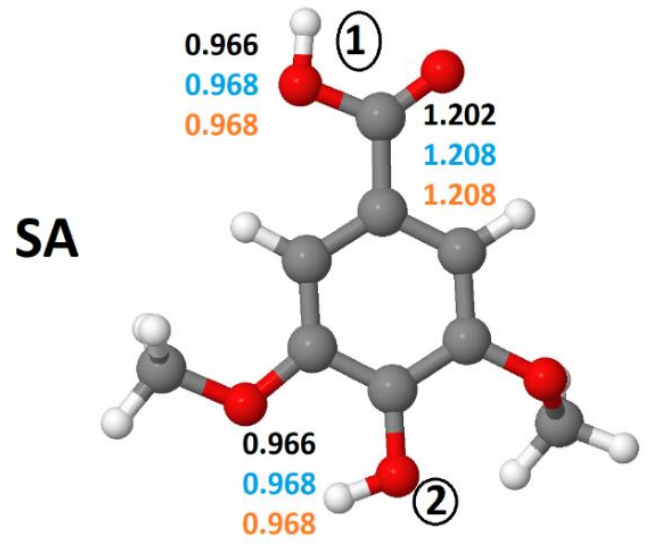

PHBA

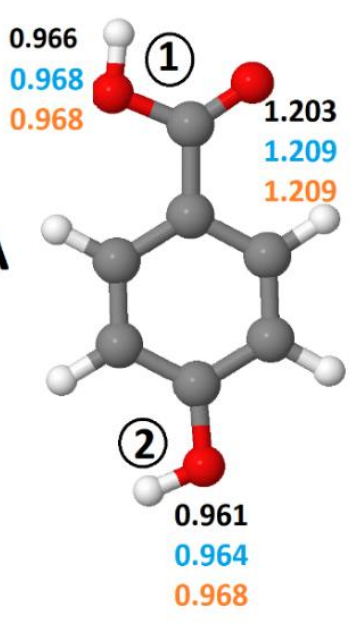

Water

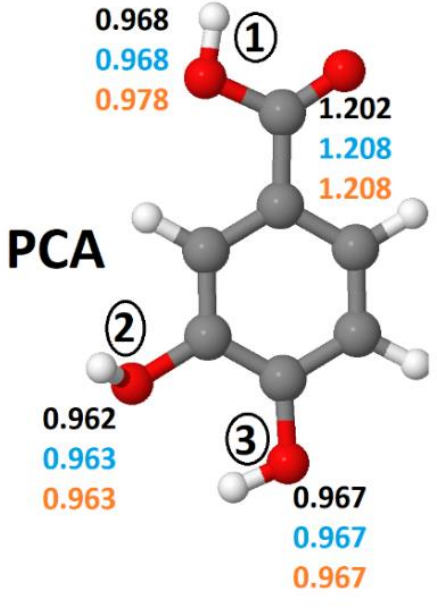

Methanol

VA

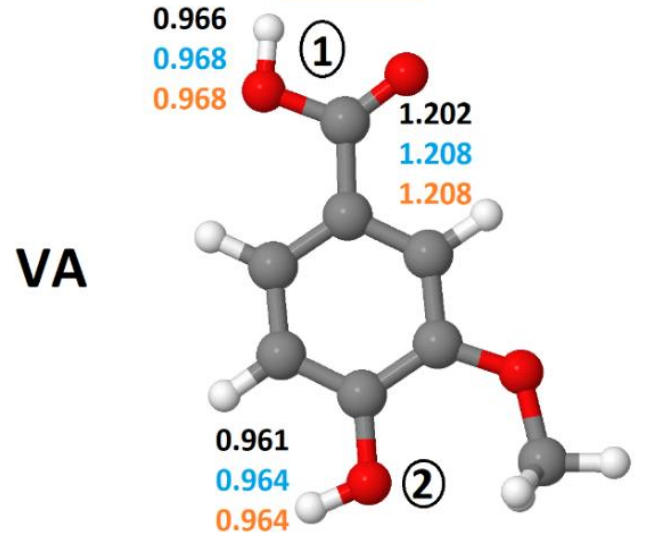

Figure 1. Optimized geometries of the antioxidants considered in this study. Bond lengths in $\AA$ computed at the M06-2X/6-311++G(d,p) level 
In the present study, we report an in-silico investigation of the antioxidant activity of five BADs using the M06-2X and B3LYP functionals combined with the 6-31++G(d,p) and 6-311++G(d,p) basis sets. As shown in Figure 1, these BADs comprise gallic acid (GA), para-hydroxybenzoic acid (PHBA), protocatechuic acid (PCA), syringic acid (SA), and vanillic acid (VA). All of these compounds exist naturally in plant food as components of complex structures like lignins and hydrolysable tanins, or attached to cell walls and proteins [29][30]. Although their antioxidant activity had been the subject of numerous experimental works [31][32][33], it was time to provide further theoretical support to the empirical data accumulated so far by responding to two main questions: what is the order of antioxidant power of these structurally related molecules with respect to either of the HAT, SET-PT and SPLET mechanisms? How can we predict the most active site of each molecule? Recall that gallic acid is assumed to be the most active of the lot. This molecule, nicknamed the molecular rival of cancer by Verma et al [34], is abundantly found in tomato [35], grapes, berries and several other fruits [36][37]. It is also one of the constituents of several hard wood plant species [38][39]. Apart from its antioxidant activity, gallic acid and its derivatives also exhibit anti-inflammatory [40], anti-mutagenic [41] and anticancer activities [42]. To account for the strong dependence of antioxidants properties on the medium where the system is found, we consider in this study three environments, i.e. gas phase, water and methanol solutions. We begin the investigation by giving a small talk on the optimized geometries of the five antioxidants as suggested by the M06-2X/6-311++G(d,p) level. Then, their antioxidant properties are compared based on thermodynamics parameters derived from the HAT, SET-PT and SPLET mechanisms. The data collected is then examined to postulate on the most preferred pathway in gas phase and polar solutions, as well as the most plausible order of antioxidant activity in each medium. We have also attempted to understand the effect of the $\mathrm{H}$ abstraction on the aromaticity of the benzene ring in terms of changes of bond lengths, using the Harmonic Oscillator Model of Aromaticity (HOMA). Finally, the reactivity of these molecules towards free radicals is debated using frontier molecular orbitals and the fukui function $\mathrm{f}^{0}$. All these tools are known to be very effective in elucidating the reactivity of molecular systems. 


\section{Computational details}

All the structures, i.e. neutral molecules, radicals, cations, and anions, were fully optimized using the meta hybrid M06-2X functional in conjunction with the $6-311++G(d, p)$ basis set as implemented in Gaussian 09 software [43]. The choice of the M06-2X/6-311++G(d,p) level of theory was motivated by the fact that it has been shown to provide reliable results about the antioxidant activities of many families of compounds [22][44]. Vibrational frequency calculations confirmed the predicted stationary point as being real minima on the potential energy surface. Bond dissociation enthalpies (BDE), ionization potentials (IPs), Proton Affinities (PA), proton dissociation enthalpies (PDEs) and electron transfer enthalpies (ETEs) were calculated using equations 1-5, where Ar-OH is the neutral form of the antioxidant, $\mathrm{ArO}^{\bullet}$ its $\mathrm{H}$ abstracted radical, $\mathrm{ArO}^{+\bullet}$ and $\mathrm{ArO}^{-}$its radical cation and anion. Formation enthalpies for the proton and electron in gas phase were fixed at $1.483 \mathrm{kcal} / \mathrm{mol}$ and $0.752 \mathrm{kcal} / \mathrm{mol}$ as obtained by Bartmess through numerical solution of Fermi-Dirac statistical mechanics equations [45]. Their solvation enthalpies in water and methanol at the M06-2X/6-311++G(d,p) level were retrieved from a benchmarking study by Rimarčík and coworkers [46]. Note that solvent contributions to the total enthalpies were accounted for using the integral equation formalism IEF-PCM method [47][48] with default settings at $298.15 \mathrm{~K}$ and 1 atmosphere. This solvation model is not only computationally affordable, but also known to provide acceptably accurate results [49].

$$
\begin{aligned}
& B D E=H\left(\mathrm{ArO}^{\bullet}\right)+H\left(\mathrm{H}^{\bullet}\right)-H(\mathrm{ArOH}) \\
& I P=H\left(\mathrm{ArO}^{+\bullet}\right)+H\left(\mathrm{e}^{-}\right)-H(\mathrm{ArOH}) \\
& P D E=H\left(\mathrm{ArO}^{\bullet}\right)+H\left(\mathrm{H}^{+}\right)-H\left(\mathrm{ArO}^{+\bullet}\right) \\
& P A=H\left(\mathrm{ArO}^{-}\right)+H\left(H^{+}\right)-H(\mathrm{ArOH}) \\
& E T E=H\left(\mathrm{ArO}^{\bullet}\right)+H\left(e^{-}\right)-H\left(\mathrm{ArO}^{-}\right)
\end{aligned}
$$


The Harmonic Oscillator Model of Aromaticity (HOMA) is used to assess the local aromaticity of the neutral and radicals forms of GA, PHBA, PCA, SA and VA. HOMA is a structural aromaticity index defined by Kruszewski and Krygowski [50] based on the idea that, in aromatic rings, bonds tend to equalize their lengths. Despite some criticism around this descriptor, many researchers have relied on it to investigate the aromaticity of a variety of system such as polycyclic aromatic hydrocarbons and fullerenes [50]. In the present case, equation 6 is employed to estimate the HOMA, with $n=6$ the number of bonds forming the ring, an empirical constant equal to 257.7 for $\mathrm{C}-\mathrm{C}$ bonds and $\mathrm{R}_{\mathrm{opt}}$ an optimal bond length valued at $1.388 \AA$ and chosen so that HOMA varies between 0 and 1 .

$$
H O M A=1-\frac{\alpha}{n} \sum_{i=1}^{n}\left(R_{o p t}-R_{i}\right)^{2}
$$

To understand the local and global reactivity of the five benzoic acid derivatives, their frontier molecular orbitals and the free radical form of the fukui function $\mathrm{f}^{0}$ were computed at the B3LYP/6$31+\mathrm{G}(\mathrm{d}, \mathrm{p})$ level. Be reminded that $\mathrm{f}^{0}$ is a conceptual DFT descriptor estimated as the arithmetic average of the electrophilic $\mathrm{f}^{-}(\mathrm{r})$ and nucleophilic $\mathrm{f}^{+}(\mathrm{r})$ fukui functions proposed by Parr et al to quantify the response of a molecular system to the fluctuation of its electron density with respect to the number of electrons [51]. The radical fukui function has been employed in many previous studies to unveil the local reactivity of molecular systems towards radical attacks. Typical examples include the prediction of the preferred free radical binding sites of some lignins precursors [52] and the innate $\mathrm{C}-\mathrm{H}$ radical functionalization sites on multi-nitrogen containing fused arenes [53].

All the structures and molecular orbitals were visualized using Jmol [54] and VMD [55]. Isosurfaces of radical fukui functions were generated using the MultiWFN code [56], while atomic condensed values were calculated using NBO charges [57]. 


\section{Results and discussion}

\subsection{Geometry and local aromaticity}

Figure 1 presents optimized geometries of neutral molecules in gas phase, water and methanol solutions, as well as the convention used herein to differentiate the $-\mathrm{OH}$ groups of each molecule. It also indicates $\mathrm{O}-\mathrm{H}$ and $\mathrm{C}=\mathrm{O}$ bond lengths predicted in the three environments. Owing to the distinct electronic surroundings of $\mathrm{OH}$ groups in these molecules, they should exhibit different reactivity towards free radicals. On this matter, most studies agree on the fact that the greatest reactivity of benzoic acid derivatives is held in the $-\mathrm{OH}$ group in para position of the carboxylic group [58]. In section 3.2, this question is re-examined. For now, let us first look at the geometry of these molecules. Figure 1 shows that carboxylic O-H bonds $\mathbf{1}$ are the longest, measuring between 0.996 and $0.978 \AA$ in the gas phase, and between 0.968 and $0.978 \AA$ in polar solution. The longest O-H bonds $\mathbf{1}$ are found in PCA regardless of the environment. Notice also that meta-positioned $\mathrm{O}-\mathrm{H}$ bonds are slightly shorter than para ones. Furthermore, the length of $\mathrm{C}=\mathrm{O}$ bonds changes from 1.202-1.203 $\mathrm{A}$ to $1.208-1.209 \AA$ when going from gas phase to polar solutions $\left(\mathrm{H}_{2} \mathrm{O}\right.$ and $\left.\mathrm{MetOH}\right)$. Altogether, these structural features suggest that both the $\mathrm{O}-\mathrm{H}$ and $\mathrm{C}=\mathrm{O}$ are respectively subjected to elongations of up to $0.010 \AA$ and $0.006 \AA$ when these molecules are dissolved in water or methanol. This observation makes sense because, even in the more realistic situation of explicit solvation where water or methanol molecules would be filled in a cage containing each of these antioxidants, one should expect intermolecular $\mathrm{O}-\mathrm{H} \ldots \mathrm{O}$ and $\mathrm{C}=\mathrm{O} \ldots \mathrm{H}$ hydrogen bonds to be formed between the solute and solvating molecules, resulting in similar bond stretching [59]. However, the computational cost associated with such simulations constitute a limiting factor. 


\subsection{HAT mechanism}

Table 2 collects calculated BDEs of the five antioxidants in gas, $\mathrm{H}_{2} \mathrm{O}$, and MetOH phases. These BDEs range between 78.2 to $111.9 \mathrm{kcal} / \mathrm{mol}$, whose magnitude accords with previous theoretical studies on similar systems [60][61]. In the gas phase, the lowest BDE is associated with the OH group $\mathbf{3}$ of PCA, while the highest corresponds to the carboxylic OH group 1 of VA. Based on lowest BDEs values, one can advance the following order of antioxidant activity in the gas phase: $\mathrm{PHBA}<\mathrm{SA}<\mathrm{VA}<\mathrm{GA}<\mathrm{PCA}$. In addition, notice that the $\mathrm{OH}$ group in para position exhibits the lowest BDEs in all the compounds and should therefore be the most active in the gas phase through the HAT pathway. Turning now on the results in solution, Table 1 reveals that the hydrogen abstraction ability of these compounds is only slightly affected by the two polar solvents, with deviations representing less than $10 \%$ of the dissociation enthalpy in the gas phase. This observation agrees well with previous findings on the antioxidant activity of benzoic acid derivatives [22]. Moreover, GA appears to be the most active in $\mathrm{H}_{2} \mathrm{O}$ and $\mathrm{MetOH}$ solutions, with the lowest BDE of 78.2 and $80.4 \mathrm{kcal} / \mathrm{mol}$ respectively in water and methanol attributed to the $\mathrm{OH}$ group 3. These values corroborate with experimental data reported by Denisova et al [62], and Alberti et al [63], who found BDEs of 83.03 and $81.00 \mathrm{kcal} / \mathrm{mol}$ in methyl linoleate and acetonitrile/chlorobenzene respectively. In polar solution, the trend of antioxidant activity is slightly different as GA gets more active than PCA $[\mathrm{PHBA}<\mathrm{SA}<\mathrm{VA}<\mathrm{PCA}<\mathrm{GA}]$. In all the three environments, PHBA is predicted as the least active if the HAT mechanism is concerned. 
Table 1. Bond dissociation enthalpies (BDE in $\mathrm{kcal} / \mathrm{mol}$ ) of the investigated benzoic acid derivatives in vacuum and solution (water and methanol) were computed at M06-2X/6-311++G(d, p) level.

\begin{tabular}{|c|c|c|c|c|c|}
\hline Antioxidant & Medium & O-H(1) & O-H(2) & O-H(3) & O-H(4) \\
\hline \multirow{3}{*}{ GA } & gas & 110.8 & 88.0 & 80.2 & 80.3 \\
\hline & water & 105.1 & 83.8 & 78.2 & 80.7 \\
\hline & methanol & 107.9 & 86.1 & 80.4 & 82.8 \\
\hline \multirow{3}{*}{ PHBA } & gas & 110.6 & 88.1 & & \\
\hline & water & 110.8 & 87.8 & & \\
\hline & methanol & 121.4 & 89.9 & & \\
\hline \multirow{3}{*}{ PCA } & gas & 110.8 & 87.4 & 78.8 & \\
\hline & water & 105.1 & 83.7 & 79.4 & \\
\hline & methanol & 107.8 & 86.0 & 81.5 & \\
\hline \multirow{3}{*}{$\mathbf{S A}$} & gas & 110.5 & 83.5 & & \\
\hline & water & 104.1 & 84.4 & & \\
\hline & methanol & 106.8 & 86.4 & & \\
\hline \multirow{3}{*}{ VA } & gas & 111.9 & 80.7 & & \\
\hline & water & 104.6 & - & & \\
\hline & methanol & 109.2 & 85.7 & & \\
\hline
\end{tabular}

Although BDEs values clearly point out the most active site and compounds in gas (and polar phases), they do not tell why this should be so. Fortunately, common sense suggests that, after homolytic breakage of an $\mathrm{O}-\mathrm{H}$ bond, the single electron left on the $\mathrm{O}$ atom delocalizes over the molecule and one can assume that the more it spreads away from its initial position, the higher the stability of the radical and the lower should be the BDE. Therefore, as a first approach, it is possible to rely on the distribution of spin densities to interpret the trends in BDE values [64]. In our present case, spin density distributions of the radicals were calculated at the M06-2X/6-311++G $(\mathrm{d}, \mathrm{p})$ level and are here listed in Table 2. Inspection of this table suggests that for the same compound, the OH group in para position has the lowest spin density estimated to $0.66,0.67 .0 .68 .0 .71$ and 0.61 respectively for GA, PHBA, PCA, SA and VA, and should lead to the most stable radical. This observation is consistent with the BDEs listed in Table 1. Similarly, the highest spin density of $0.96-0.98$ predicted for the carboxylic $\mathrm{OH}$ group corroborate well with the high BDEs reported in Table 1. 
Table 2. Local spin density calculated on the $\mathrm{O}$ atom after the homolytic cleavage of $\mathrm{O}-\mathrm{H}$ bond in the gas, $\mathrm{H}_{2} \mathrm{O}$ and $\mathrm{MetOH}$ phases. Values calculated at the M06-2X/6-311++G(d,p) level.

\begin{tabular}{lllll}
\hline Antioxidant & $\mathbf{O ( 1 )}$ & $\mathbf{O}(\mathbf{2})$ & $\mathbf{O}(\mathbf{3})$ & $\mathbf{O ( 4 )}$ \\
\hline GA & 0.97 & 0.70 & 0.66 & 0.68 \\
PHBA & 0.97 & 0.67 & & \\
PCA & 0.96 & 0.70 & 0.68 & \\
SA & 0.98 & 0.71 & & \\
VA & 0.97 & 0.61 & & \\
\hline
\end{tabular}

Some studies have shown that the stability order of $\mathrm{H}$ abstraction radicals can be regarded as the consequence of the number of resonance forms pointing to the same particular hybrid, assuming the conjugation contribution to be essentially the same for all the considered systems [65][66]. However, you will admit with us that counting resonance structures is not a reliable nor systematic approach. One thing is sure, once the radical is formed, its unpaired electron delocalizes over the benzene ring. This redistribution of the internal electron density produces considerable structural changes, which affect the original aromaticity of the ring. To test this assumption (in gas phase) and find the link between the stability of $\mathrm{H}$ abstraction radicals and the change in aromaticity, we have computed HOMA values related to the benzene ring in parent molecules and all the radicals originating from the removal of one $\mathrm{H}$ atom (Figure 2). As shown in Figure 2, HOMAs are comprised between 0.980 and 0.993 for parent molecules, which denotes strong aromaticity. When an $\mathrm{H}$ atom is removed from one of the $\mathrm{O}-\mathrm{H}$ groups, these values generally decrease and denotes the loss of atomicity by the ring. More importantly, the higher the dearomatization, the higher the stability of the radical. Therefore, the induced dearomatization is the most pronounced when the $\mathrm{H}$ atom is expelled from the $\mathrm{O}-\mathrm{H}$ group in para position. For instance, in the case of GA, radical 3 has the lowest HOMA of 0.499, followed by radical 4 (O.542), radical $2(0.623)$ and radical 1 (0.993), while the order of stability of these radicals is as follows: radical $3>$ radical $4>$ radical $2>$ radical 1 . This paradoxical observation may be understood in terms of compensation: radicals being naturally instable, the loss of aromatic stability tries to compensate over its intrinsic instability. All these observations seems to go in the same direction as the findings reported in a recent study (2021) by Lin $e t$ 
al who used various aromaticity induces to systematically investigate the relationship between aromaticity and the thermodynamic stability of $\alpha$-methyl heterocyclics [67]. In sharp contrast with the general knowledge that aromaticity is the carrier of thermodynamic stability, their calculations showed that the stronger the antiaromaticity of the original form heterocyclics, the higher the thermodynamic stability of the corresponding radicals.

However, it must be noted that the previous rule (i.e. the higher the dearomatization, the more stable the radical) as well as the analysis of spin densities are only useful when it comes to predicting the most active site within the same molecule, and do not have to be extended to a whole dataset of systems. To illustrate this, let us consider VA and PCA. Figure 2 shows that the HOMA of radical 2 of VA is 0.404 while that for radical 3 of PCA is 0.418 . In contrast, the homolytic rupture of the $\mathrm{H}$ atom from $\mathrm{O} 3-\mathrm{H}$ group of PCA requires less energy than the one needed to split-off O2-H in VA. Similarly, spin densities at $\mathrm{O} 3$ (PCA) and $\mathrm{O} 2(\mathrm{VA})$ are respectively 0.67 and 0.61 , while corresponding BDEs are estimated to 78.8 and $80.7 \mathrm{kcal} / \mathrm{mol}$ respectively in gas phase. Similar conclusions can be made in solutions. In section 3.6, we have addressed the same problem from a completely different angle, that of predicting the $\mathrm{O}$ atom that is the most likely to interact with an approaching free radical. 

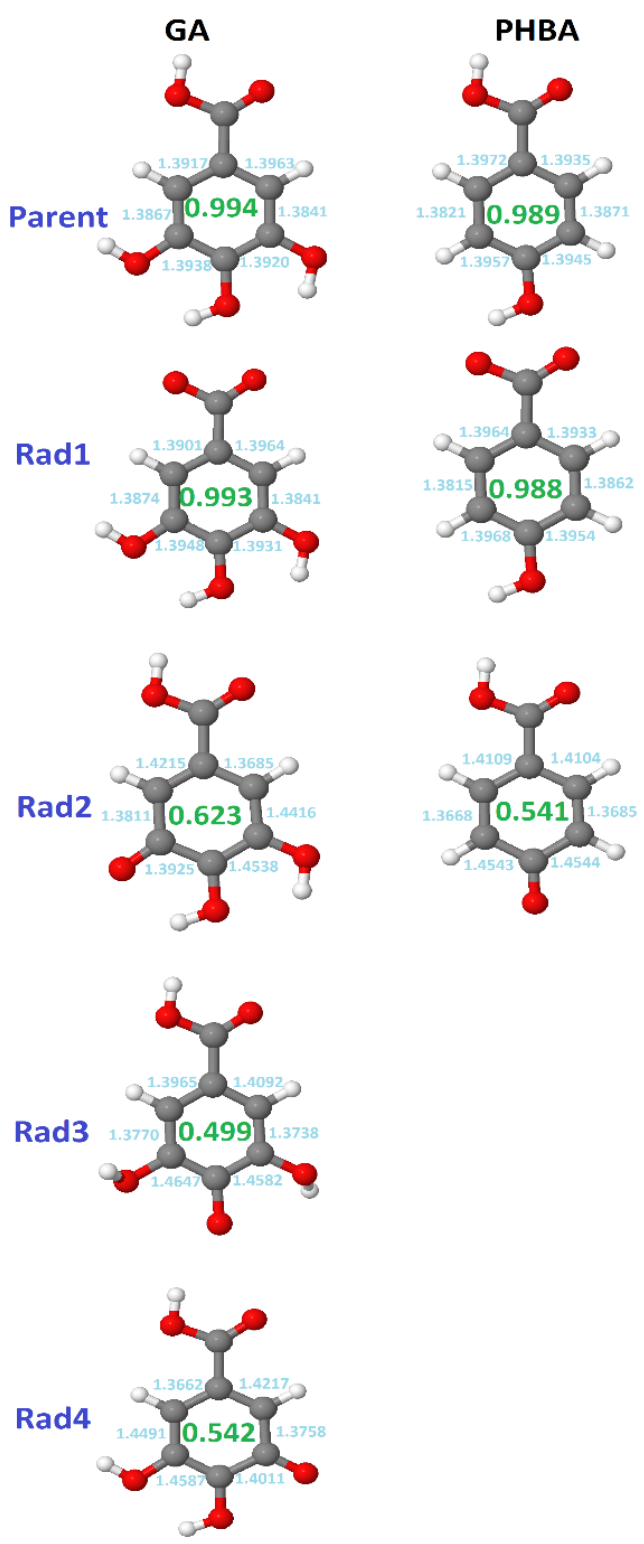
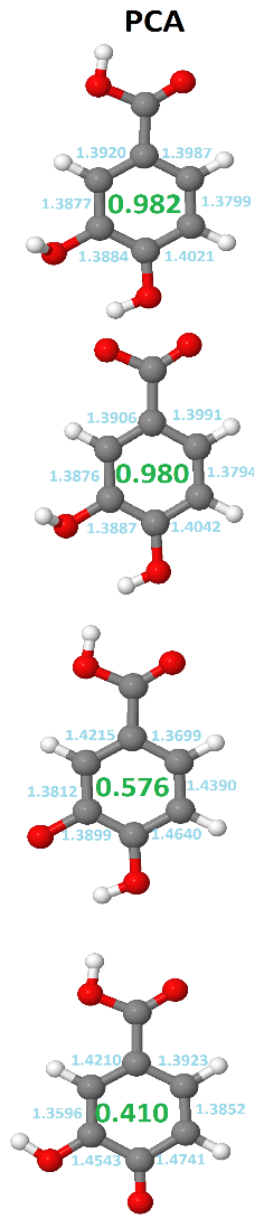

HOMA

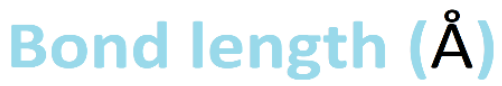

Figure 2. HOMA and bond lengths of the parent and radical resulting from the homolytic rupture of OH bonds from GA, PHBA, PCA, SA and VA. 


\subsection{SET-PT mechanism}

Table 3 reports the calculated IPs and PDEs of the five antioxidants in the gas phase and $\mathrm{H}_{2} \mathrm{O}$ and MetOH solutions at the M06-2X/6-311++G(d,p) level. The data in this table reveal that the IP values range between 185.5 and $195.5 \mathrm{kcal} / \mathrm{mol}$ in gas phase, and between 121.8 and $132.9 \mathrm{kcal} / \mathrm{mol}$, and 127.1 and $138.3 \mathrm{kcal} / \mathrm{mol}$ respectively in water and methanol. These values are in the same range as those obtained in previous studies [65][68]. As far as the SET-PT model is concerned, lowest IPs values suggest the following orders of antioxidant activity: PHBA $<$ PCA GA $<$ VA $<$ SA, PHBA $<$ GA $\sim$ PCA $<$ VA $<$ $\mathrm{SA}$ and $\mathrm{PHBA}<\mathrm{VA}<\mathrm{GA} \sim \mathrm{PCA}<\mathrm{SA}$ respectively in gas phase, $\mathrm{H}_{2} \mathrm{O}$ and methanol. These patterns are far different from the one obtained with BDEs. In order words, if the reaction had to follow this pathway, then the order of antioxidant activity should be different from the one inferred from the analysis of BDEs. This apparent discrepancy between IPs and BDEs is not surprising. It may related to the fact BDEs are local quantities depending on the relative positions of substituents, whereas IPs are global parameters affected by the structure of the entire molecule [69]. Furthermore, it is worth noting that IPs decrease drastically from the gas phase to polar media. For instance, the IP of SA plummets from 185.5 to 124.6 $\mathrm{kcal} / \mathrm{mol}$ on going from the gas phase to aqueous solution. This shows that polar solvents considerably facilitate the electron transfer to the free radicals and thus enhance the antioxidant activity. This result is in agreement with many documented studies on antioxidant properties [70]. Notice also that IP values follow the order water $<$ methanol $<$ gas, which indicates that the stronger the solvent polarity, the higher the electron-donating ability of the molecule [71]. 
Table 3. Ionization potential (IP) and proton dissociation enthalpy (PDE) computed at the M06-2X/6$311++\mathrm{G}(\mathrm{d}, \mathrm{p})$ level. PDE values are provided for each site. Values are given in $\mathrm{kcal} / \mathrm{mol}$.

\begin{tabular}{|c|c|c|c|c|c|c|}
\hline \multirow{2}{*}{ Antioxidant } & \multirow[t]{2}{*}{ Medium } & \multirow[t]{2}{*}{ IP } & \multicolumn{4}{|c|}{ PDE } \\
\hline & & & O1-H & O2-H & O3-H & O4-H \\
\hline \multirow{3}{*}{ GA } & vacuum & 194.4 & 232.3 & 201.6 & 201.8 & 209.5 \\
\hline & water & 124.8 & 27.9 & 3.6 & 1.0 & 6.5 \\
\hline & methanol & 130.1 & 23.9 & -1.2 & -3.6 & 2.0 \\
\hline \multirow{3}{*}{ PHBA } & vacuum & 204.2 & 222.3 & 199.8 & & \\
\hline & water & 132.9 & 2.4 & 2.4 & & \\
\hline & methanol & 138.3 & 29.3 & -2.3 & & \\
\hline \multirow{3}{*}{ PCA } & vacuum & 195.2 & 231.5 & 208.1 & 199.5 & \\
\hline & water & 124.6 & 28.0 & 6.7 & 2.4 & \\
\hline & methanol & 129.9 & 24.0 & 2.1 & -2.3 & \\
\hline \multirow{3}{*}{ SA } & vacuum & 185.5 & 240.9 & 213.9 & & \\
\hline & water & 121.8 & 29.7 & 10.4 & & \\
\hline & methanol & 127.1 & 25.8 & 5.5 & & \\
\hline \multirow{3}{*}{ VA } & vacuum & 189.6 & 238.2 & 207.1 & & \\
\hline & water & 122.5 & 29.7 & 7.0 & & \\
\hline & methanol & 134.1 & 19.2 & -4.2 & & \\
\hline
\end{tabular}

The proton dissociation enthalpy (PDE) characterizes the second step of the SET-PT mechanism and serve to decipher the thermodynamically preferred $\mathrm{OH}$ group for deprotonation from the cation radical. It can be seen from Table 3 that the lowest PDE in all the three environments is associated to the $\mathrm{OH}$ group in para position and agrees well with the results based on BDEs. Moreover, PCA has the lowest PDE in gas phase, while this applies to GA in polar solution. Like IP values, there are substantial decrease of PDEs in solution as compared to the gas phase, which may be ascribed to the high stabilization of the positively charged proton in polar solution. Note that, in gas phase, the lowest PDEs suggest the following order of antioxidant activity SA $<\mathrm{VA}<\mathrm{GA}<\mathrm{PHBA}<\mathrm{PCA}$, which is slightly different from the one obtained in solution, i.e SA $<$ VA $<$ PHBA $<$ PCA $<$ GA. The negative PDE values predicted for some $\mathrm{OH}$ groups may suggest a plausible mediation of the solvent in the heterolytic breakage of the O-H bonds of the radical cation, pulling and favoring the ejection of the proton with no need for extra energy. Wang 
et al reported similar data in their investigation of the antioxidant activity of genistein and its nitro and amino derivatives [72].

\subsection{SPLET mechanism}

Table 5 presents proton affinities (PAs) and electron transfer enthalpies (ETEs) of GA, PHBA, PCA, SA and VA in the gas phase and polar $\left(\mathrm{H}_{2} \mathrm{O}\right.$ and MetOH) solutions at the M06-2X/6-311++G(d,p) level. In gas phase, PA values are comprised between 329.2 and $344.0 \mathrm{kcal} / \mathrm{mol}$, the lowest and highest boundaries being associated with the $\mathrm{O} 2-\mathrm{H}$ and $\mathrm{O} 4-\mathrm{H}$ groups of GA. It must be noted that, in gas phase, the $\mathrm{O} 2-\mathrm{H}$ bond has the lowest PA and should be the most likely to undergo a heterolytic breakage. This group correspond to the para position with respect to the carboxylic group, except for GA (meta). In polar solution, this applies to the $\mathrm{O} 1-\mathrm{H}$ bond for all the molecules, except for GA in water where O4-H has the lowest PA of $30.5 \mathrm{kcal} / \mathrm{mol}$. Inspection of Table 4 reveals that PAs drop considerably when going from gas phase to polar solution (where PA values are lower than $39.1 \mathrm{kcal} / \mathrm{mol}$ ), following the order gas phase $<<$ water $<$ methanol. This pattern can be related to the high stability of the radical anion and proton in solution as compared to the gas phase. Considering the lowest PA values in gas phase, one can deduce the following order of antioxidant activity $\mathrm{PHBA} \sim \mathrm{SA}<\mathrm{VA}<\mathrm{PCA}<\mathrm{GA}$ in gas phase, which changes to $\mathrm{PHBA} \sim \mathrm{VA}<\mathrm{PCA}<\mathrm{SA}<\mathrm{GA}$ in polar medium. 
Table 4. Proton affinity (PA) and electron transfer enthalpy (ETE) computed at the M06-2X/6$311++\mathrm{G}(\mathrm{d}, \mathrm{p})$ level. ETE values are provided for each site. Values are given in $\mathrm{kcal} / \mathrm{mol}$.

\begin{tabular}{|c|c|c|c|c|c|c|c|c|c|}
\hline \multirow[t]{2}{*}{ Antioxidant } & \multirow[t]{2}{*}{ Medium } & \multicolumn{4}{|c|}{$\mathbf{P A}$} & \multicolumn{4}{|c|}{ ETE } \\
\hline & & $\begin{array}{c}\text { O1- } \\
\text { H }\end{array}$ & $\begin{array}{c}\text { O2- } \\
\text { H }\end{array}$ & $\begin{array}{c}\text { O3- } \\
\text { H }\end{array}$ & O4-H & $\begin{array}{c}\text { O1- } \\
\text { H }\end{array}$ & $\begin{array}{c}\text { O2- } \\
\text { H }\end{array}$ & $\begin{array}{c}\text { O3- } \\
\text { H }\end{array}$ & $\begin{array}{c}\text { O4- } \\
\text { H }\end{array}$ \\
\hline \multirow{3}{*}{ GA } & vacuum & 337.3 & 329.2 & 330.3 & 344.0 & 89.4 & 67.0 & 65.9 & 59.9 \\
\hline & water & 39.1 & 31.8 & 33.2 & 30.5 & 112.0 & 95.0 & 91.1 & 99.4 \\
\hline & methanol & 29.3 & 31.7 & 30.4 & 37.9 & 124.1 & 96.7 & 95.5 & 93.6 \\
\hline \multirow{3}{*}{ PHBA } & vacuum & 339.3 & 332.5 & & & 87.2 & 71.5 & & \\
\hline & water & 31.3 & 34.4 & & & 125.5 & 99.4 & & \\
\hline & methanol & 30.2 & 32.9 & & & 136.8 & 102.5 & & \\
\hline \multirow{3}{*}{ PCA } & vacuum & 337.5 & 330.5 & 337.0 & & 89.2 & 64.3 & 66.3 & \\
\hline & water & 30.9 & 33.4 & 35.9 & & 120.2 & 92.0 & 93.8 & \\
\hline & methanol & 29.7 & 31.9 & 34.6 & & 123.6 & 95.1 & 96.9 & \\
\hline \multirow{3}{*}{ SA } & vacuum & 337.7 & 332.3 & & & 88.7 & 67.8 & & \\
\hline & water & 30.6 & 35.9 & & & 119.5 & 94.5 & & \\
\hline & methanol & 29.4 & 34.5 & & & 122.9 & 97.4 & & \\
\hline \multirow{3}{*}{ VA } & vacuum & 338.6 & 331.8 & & & 89.1 & 58.0 & & \\
\hline & water & 31.0 & 36.4 & & & 119.7 & 91.6 & & \\
\hline & methanol & 30.1 & 35.2 & & & 123.2 & 94.7 & & \\
\hline
\end{tabular}

After proton dissociation, the next step of the SPLET mechanism consists in the transfer of an electron from $\mathrm{ArO}^{-}$to the free radical. The capability to release this electron density, which is measured by the electron transfer enthalpy (ETE), tells how easily the anionic form of the antioxidant completes this step. ETE values listed in Table 4 are in between 58.0 and $89.4 \mathrm{kcal} / \mathrm{mol}$ in gas phase and 91.1 and $136.8 \mathrm{kcal} / \mathrm{mol}$ in polar solution. In gas phase, site $\mathrm{O} 2$ has the lowest ETE value, except in the case of GA for which it is site $\mathrm{O} 4$ instead. As such, one can expect these sites (O2 for all, except GA) to be enriched in extra electron density by a striking free radical. Moreover, looking at the lowest ETE values in gas phase, the anionic form of VA appears to be the ablest to trap an electron from a free radical, followed respectively by that of GA, PCA, SA and PHBA. 
As opposed to PAs, ETE values increase in solution, but their increase is less pronounced than the associated decrease of PA values. Thus, the overall effect in a decrease in the energy needed to pass the second step as compared to the gas phase. This finding can be explained by the high solubility of the radical anion $\left(\mathrm{ArO}^{-}\right)$in polar solvents. Let us note that site $\mathrm{O} 2$ has the lowest ETE in both solvents for all compounds except GA, for which site O3 and O4 come up with the lowest ETE values of 91.1 and 93.6 $\mathrm{kcal} / \mathrm{mol}$ respectively in water and methanol respectively. In line with the lowest ETE values, the electron transfer ability of these compounds is as follows: PHBA $<$ SA $<$ PCA $<$ VA $\sim$ GA in both water and methanol and is very close to that observed in gas phase.

\subsection{Thermodynamically preferred mechanism}

To figure out the preferred mechanistic pathway of antioxidant activity, several researchers have relied on the comparison of thermodynamics parameters characterizing the first step of each mechanism. [44][73]. The same approach is here applied. Recall that IP and PA values describe the first step of the SET-PT and SPLET mechanisms, while BDEs describe the unique reaction of the HAT mechanism. Looking at tables 2,4-5, we notice that BDEs are substantially smaller than IPs and PAs in gas phase. This suggests that the antioxidant activity of GA, PHBA, PCA, SA and VA is dictated by the HAT mechanism in gas phase and corroborates with Urbaniak et al [74]. As such, PCA and PHBA should display respectively the highest and lowest antioxidant activity in gas phase. Furthermore, Tables 2, 4-5 reveal that, in polar solution, PAs are very low as compared to BDEs and IPs. This finding confirms the SPLET mechanism as being the most favorable in water and methanol. Therefore, GA and PHBA are respectively the most and least active in polar medium. Note that, regardless of the environment, PHBA seems to be the least active of the set. 


\subsection{Reactivity}

\subsubsection{Frontier molecular orbitals}

Frontier molecular orbitals (FMOs) are widely used to probe the reactivity of molecular systems [75]. Most often, one can rely on the electronic density distribution in these orbitals to predict the most preferred attack site for free radicals and other reactive agents [73]. More interestingly, previous studies have shown that the free radical scavenging ability of phenolic compounds can be related to the energy of the HOMO such that molecules with higher HOMO energies are likely to be more active due to the stronger electron donating ability [44][73]. To check this "HOMO-rule" and assess the local reactivity of the compounds considered here, FMOs of the five antioxidants were computed in the three environments. Figure 3 displays the electron density distribution of the FMOs of our antioxidants in gas phase (qualitatively equivalent plots were obtained in solution), as well as the corresponding energies. Electronic energies associated with the HOMO and LUMO in gas phase and $\mathrm{H}_{2} \mathrm{O}$ and MetOH solutions are provided in Table 6.

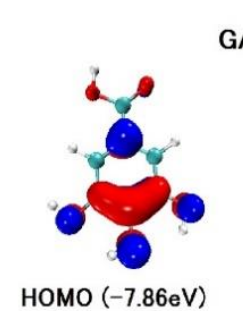

GA

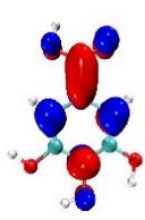

LUMO $(-0.55 \mathrm{eV})$

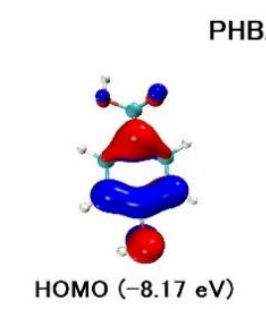

PHBA
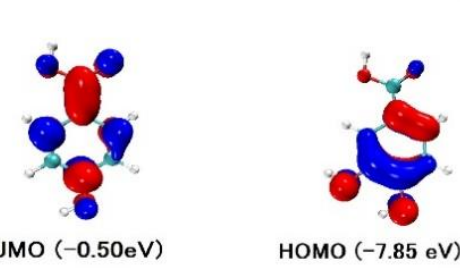

PCA

SA

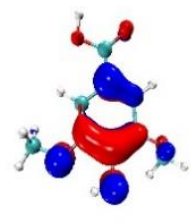

HOMO $(-7.72 \mathrm{eV})$

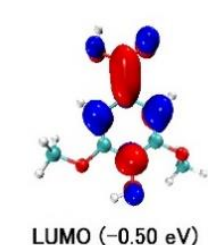

LUMO $(-0.50 \mathrm{eV})$

LUMO $(-0.50 \mathrm{eV})$

VA

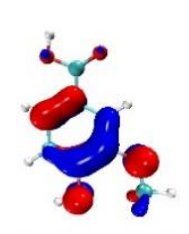

Hомо $(-7.60 \mathrm{eV})$
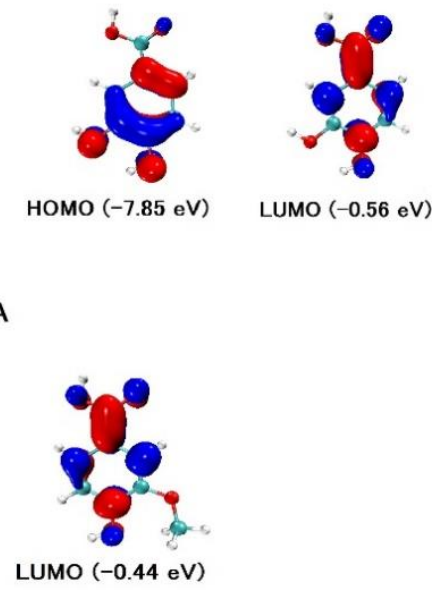

LUMO $(-0.44 \mathrm{eV})$

Figure 3. Frontier molecular orbitals of GA, PHBA, PCA, SA and VA in gas phase at the M06-2X/6$311++\mathrm{G}(\mathrm{d}, \mathrm{p})$ level. 
Figure 3 shows that the electron densities of the HOMO and LUMO are delocalized over a big portion of these molecules. The concentration of this density on $\mathrm{O}$ atoms and $\mathrm{C}$ atoms of the benzene ring suggests these atoms to be the preferred attack site for free radicals [72]. However, the reactions on $\mathrm{C}$ and $\mathrm{C}=\mathrm{O}$ sites has not been considered and may constitute the core of a separate study. From Table 6, HOMO energies are comprised between -7.60 and $-7.86 \mathrm{eV}$ in gas phase and range from -7.81 to $-8.19 \mathrm{eV}$ in polar solutions. In the gas phase, VA and PHBA have respectively the highest and lowest HOMO energies estimated to -7.60 and $-8.17 \mathrm{eV}$. Assuming the validity of the "HOMO-rule", VA should be the strongest electron donor in the gas phase and PHBA the weakest. Applying the same reasoning in polar phase leads to postulate SA as the best electron donor with the highest HOMO energy of $-7.81 \mathrm{eV}$. However, when we compared HOMO energies listed in Table 6, we deduced the following orders of free electron donor ability: $\mathrm{PHBA}<\mathrm{GA} \sim \mathrm{PCA}<\mathrm{SA}<\mathrm{VA}$ and $\mathrm{PHBA}<\mathrm{VA}<\mathrm{GA}<\mathrm{PCA}<\mathrm{SA}$ in gas and polar phases respectively, which do not corroborate with any of the antioxidant activity patterns predicted based on either of BDEs, IPs, PDEs, PAs and ETEs. This observation is paradoxical and may indicate that the HOMO rule is not absolute and may be misleading in the discussion of the antioxidant activity of some molecules. It is then advised to be cautious with it.

Table 5. Frontier molecular orbitals (FMOs) energies computed in gas phase and polar $\left(\mathrm{H}_{2} \mathrm{O}\right.$ and $\left.\mathrm{MetOH}\right)$ solution at the M06-2X/6-311++G(d,p) level. Values are expressed in eV.

\begin{tabular}{lllll}
\hline Antioxidant & FMO & Gas & Water & Methanol \\
\hline \multirow{2}{*}{ GA } & HOMO & -7.86 & -7.89 & -7.89 \\
& LUMO & -0.55 & -0.67 & -0.67 \\
PHBA & HOMO & -8.17 & -8.19 & -8.19 \\
& LUMO & -0.50 & -0.62 & -0.61 \\
PCA & HOMO & -7.85 & -7.87 & -7.87 \\
& LUMO & -0.56 & -0.66 & -0.65 \\
SA & HOMO & -7.72 & -7.81 & -7.81 \\
& LUMO & -0.50 & -0.69 & -0.68 \\
VA & HOMO & -7.60 & -8.11 & -8.10 \\
& LUMO & -0.44 & -0.68 & -0.67 \\
\hline
\end{tabular}




\subsubsection{Fukui function $\mathrm{f}^{0}$}

In section 3.5, we showed that the HAT mechanism dictates the antioxidant activity of these molecules in the gas phase. Recall the HAT is concerned with the abstraction of a $\mathrm{H}$ atom by a reactive free radical. Therefore, it can be expected that the most reactive site towards free radicals will result in lower BDE and higher activity. To elucidate this question, we have computed the fukui function $\mathrm{f}^{0}$ of all the neutral molecules in the gas phase. Figure 4 shows isosurfaces of the fukui $\mathrm{f}^{0}$ function, as well as condensed values on atoms estimated using NBO charges. Let it be noted that other population analyses would have been used to obtain atomic charges, the Hilsfield protocol being among the most favorite [Ref]. Figure 4 displays 0.003 isosurfaces of $\mathrm{f}^{0}$ as well as condensed values on $\mathrm{O}$ atoms involved in $\mathrm{O}-\mathrm{H}$ bonds.
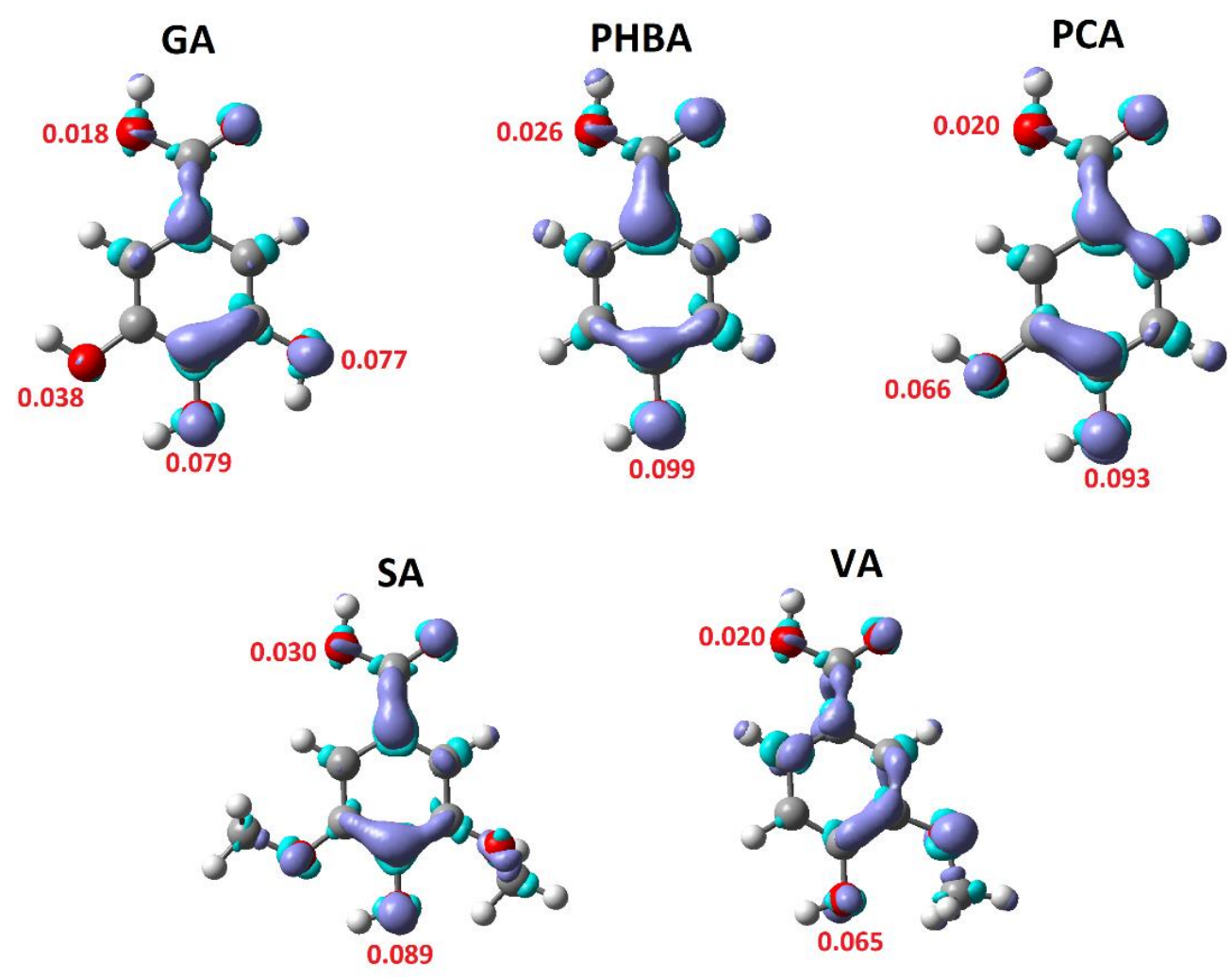

Figure 4. Isosurface plots of fukui function $\mathrm{f}^{0}$ and condensed values at hydroxylic $\mathrm{O}$ atoms. 
It is clear from Figure 4 that positive values of $\mathrm{f}^{0}$ are concentrated on $\mathrm{O}$ atoms, as well as also on the $\mathrm{C}$ atoms in alpha and para-position with respect to the carboxylic group. As aforementioned, only $\mathrm{H}$ abstractions at hydroxylic O-H groups are considered here. More interestingly, it appears that the carboxylic $\mathrm{O}$ atom has the lowest $\mathrm{f}^{0}$ value, while the highest is identified with the one in para position. This observation corroborates with the local spin densities presented in section 3.2. However, it must be highlighted that both $\mathrm{f}^{0}$ and local spin densities should be generalized to all the five systems. In other words, their predictive power should be restricted to the local reactivity of a given molecule, not never be ued to compare atomic values of different molecules.

\section{Conclusion}

The antioxidant activity of five benzoic acid derivatives has been investigated at the M06-2X/6$311++\mathrm{G}(\mathrm{d}, \mathrm{p})$ level, following three possible mechanisms of antioxidant activity in gas and polar phases. Our findings suggest that the hydrogen atom transfer (HAT) is the preferred mechanistic pathway in gas phase, while the sequential proton loss electron transfer (SPLET) is favored in polar mediums. Protocatechuic and gallic acids (PCA and GA) are the most active in gas and polar solutions respectively, whereas PHBA is the least active in all the environments considered. Solvents are found to induce considerable changes in the enthalpies of charged species, explaining some drastic variations in proton affinities (PA) and Proton Dissociation Enthalpies (PDE) when going from gas phase to polar solutions. The $\mathrm{O}-\mathrm{H}$ group in para position of the carboxylic group $(\mathrm{O} 3-\mathrm{H}$ for $\mathrm{GA}$ and $\mathrm{O} 2-\mathrm{H}$ for the rest) is the most reactive site in gas phase, while in solution it is either the O1-H site for PHBA, PCA, SA and VA, or O4$\mathrm{H}$ for GA. The Fukui function $\mathrm{f}^{0}$ seems to be a good indicator of the local reactivity of these molecules in the gas phase. Finally, we have shown that the dearomatization of $\mathrm{H}$ abstraction radicals with respect to parent molecules may serve to predict the most active site within the same molecule.

\section{Acknowledgements}


Bienfait K. Isamura is grateful to Prof Kevin Lobb at Rhodes University and the Center for High Performance Computing (CHPC) for having provided the resources used to carry out this study (project CHEM0802). He also thanks the BEBUC scholarship system for financial support.

\section{Declaration}

The authors declare no conflict of interest

\section{References}

[1] Elaka IK, Kapepula PM, Ngombe NK, et al. Microscopic Features, Antioxidant and Antibacterial Capacities of Plants of the Congolese Cosmetopoeia, Raw Materials of Cosmeceuticals. J Biosci Med 2020;08:149-166.

[2] Shahidi F. Antioxidants in food and food antioxidants. Nahrung - Food 2000;44:158-163.

[3] Patrick L. Mercury toxicity and antioxidants: Part I: Role of glutathione and alpha-lipoic acid in the treatment of mercury toxicity. Altern Med Rev 2002;7:456-471.

[4] Van Acker SABE, Van Den Berg DJ, Tromp MNJL, et al. Structural aspects of antioxidant activity of flavonoids. Free Radic Biol Med 1996;20:331-342.

[5] Llano S, Gómez S, Londoño J, et al. Antioxidant activity of curcuminoids. Phys Chem Chem Phys 2019;21:3752-3760.

[6] Kähkönen MP, Heinonen M. Antioxidant activity of anthocyanins and their aglycons. J Agric Food Chem 2003;51:628-633.

[7] Kurilich AC, Juvik JA. Quantification of carotenoid and tocopherol antioxidants in Zea mays. J Agric Food Chem 1999;47:1948-1955.

[8] Krinsky NI. Carotenoids as antioxidants. Nutrition 2001;17:815-817.

[9] Winston GW. Oxidants and antioxidants in aquatic animals. Comp Biochem Physiol Part C, Comp 1991;100:173-176.

[10] Shahidi F, Zhong Y. Antioxidants from marine by-products. Woodhead Publishing Limited; 2006.

[11] Konuray G, Erginkaya Z. Antimicrobial and antioxidant properties of pigments synthesized from microorganisms. Battle Against Microb Pathog Basic Sci Technol Adv Educ Programs 2015;2732.

[12] Ren C-Y, Wu E-L, Hartmann EM, et al. Biological Mitigation of Antibiotic Resistance Gene Dissemination by Antioxidant-Producing Microorganisms in Activated Sludge Systems. Environ Sci Technol 2021;

[13] Shahidi F, Zhong Y. Measurement of antioxidant activity. J Funct Foods 2015;18:757-781.

[14] Torres de Pinedo A, Peñalver P, Morales JC. Synthesis and evaluation of new phenolic-based antioxidants: Structure-activity relationship. Food Chem 2007;103:55-61. 
[15] Tshilanda DD, Babady PB, Onyamboko DNV, et al. Chemo-type of essential oil of Ocimum basilicum L. from DR Congo and relative in vitro antioxidant potential to the polarity of crude extracts. Asian Pac J Trop Biomed 2016;6:1022-1028.

[16] Pietta PG. Flavonoids as antioxidants. J Nat Prod 2000;63:1035-1042.

[17] Scalbert A, Johnson IT, Saltmarsh M. Polyphenols: antioxidants and beyond. Am J Clin Nutr 2005;81:215-217.

[18] Pandey KB, Rizvi SI. Plant polyphenols as dietary antioxidants in human. Oxidative Med Cell Longev 2009;2:270-278.

[19] Velika B, Kron I. Antioxidant properties of benzoic acid derivatives against Superoxide radical. Free Radicals Antioxidants 2012;2:62-67.

[20] Al-Haidari RA, Al-Oqail MM. New benzoic acid derivatives from Cassia italica growing in Saudi Arabia and their antioxidant activity. Saudi Pharm J 2020;28:1112-1117.

[21] Saravanan N, Rajasankar S, Nalini N. Antioxidant effect of 2-hydroxy-4-methoxy benzoic acid on ethanol-induced hepatotoxicity in rats. J Pharm Pharmacol 2010;59:445-453.

[22] Škorňa P, Michalík M, Klein E. Gallic acid: thermodynamics of the homolytic and heterolytic phenolic O-H bonds splitting-off. Acta Chim Slovaca 2016;9:114-123.

[23] Mohajeri A, Asemani SS. Theoretical investigation on antioxidant activity of vitamins and phenolic acids for designing a novel antioxidant. J Mol Struct 2009;930:15-20.

[24] Kalita D, Kar R, Handique JG. A theoretical study on the antioxidant property of gallic acid and its derivatives. J Theor Comput Chem 2012;11:391-402.

[25] Shang Y, Zhou H, Li X, et al. Theoretical studies on the antioxidant activity of viniferifuran. New J Chem 2019;43:15736-15742.

[26] Zheng YZ, Deng G, Chen DF, et al. Theoretical studies on the antioxidant activity of pinobanksin and its ester derivatives: Effects of the chain length and solvent. Food Chem 2018;240:323-329.

[27] Alisi IO, Uzairu A, Abechi SE. Molecular design of curcumin analogues with potent antioxidant properties and thermodynamic evaluation of their mechanism of free radical scavenge. Bull Natl Res Cent 2020;44:.

[28] Vásquez-Espinal A, Yañez O, Osorio E, et al. Theoretical Study of the Antioxidant Activity of Quercetin Oxidation Products. Front Chem 2019;7:1-10.

[29] Dai J, Mumper RJ. Plant phenolics: Extraction, analysis and their antioxidant and anticancer properties. Molecules 2010;15:7313-7352.

[30] Liu Q, Luo L, Zheng L. Lignins: Biosynthesis and biological functions in plants. Int J Mol Sci 2018;19:.

[31] Manuja R, Sachdeva S, Jain A, et al. A comprehensive review on biological activities of Phydroxy benzoic acid and its derivatives. Int J Pharm Sci Rev Res 2013;22:109-115.

[32] Cikman O, Soylemez O, Ozkan OF, et al. Antioxidant activity of syringic acid prevents oxidative stress in 1-arginine-induced acute pancreatitis: An experimental study on rats. Int Surg 2015;100:891-896.

[33] Tai A, Sawano T, Ito H. Antioxidative properties of vanillic acid esters in multiple antioxidant 
assays. Biosci Biotechnol Biochem 2012;76:314-318.

[34] Verma S, Singh A, Mishra A. Gallic acid: Molecular rival of cancer. Environ Toxicol Pharmacol 2013;35:473-485.

[35] Vinkovic Vrcek I, Samobor V, Bojic M, et al. Efecto del injerto en las propiedades antioxidantes del tomate (Solanum lycopersicum L.). Spanish J Agric Res 2011;9:844-851.

[36] Ma J, Luo XD, Protiva P, et al. Bioactive novel polyphenols from the fruit of Manilkara zapota (Sapodilla). J Nat Prod 2003;66:983-986.

[37] Shahrzad S, Bitsch I. Determination of some pharmacologically active phenolic acids in juices by high-performance liquid chromatography. J Chromatogr A 1996;741:223-231.

[38] Eyles A, Davies NW, Mitsunaga T, et al. Role of Eucalyptus globulus wound wood extractives evidence of superoxide dismutase-like.pdf. For Pathol 2004;34:225-232.

[39] Murugananthan M, Raju GB, S Prabhakar. J of Chemical Tech Biotech - 2005 - Murugananthan Removal of tannins and polyhydroxy phenols by electro-chemical.pdf. J Chem Technol Biotechnol 2005;80:1188-1197.

[40] Arunkumar S, Ilango K, Manikandan RS, et al. Synthesis and anti-inflammatory activity of some novel pyrazole derivatives of gallic acid. E-Journal Chem 2009;6:123-129.

[41] Ow Y-Y, Stupans I. Gallic Acid and Gallic Acid Derivatives: Effects on Drug Metabolizing Enzymes. Curr Drug Metab 2005;4:241-248.

[42] Mirvish SS, Cardesa A, Wallcave L, et al. Induction of mouse lung adenomas by amines or ureas plus nitrite and by $\mathrm{N}$ nitroso compounds: effect of ascorbate, gallic acid, thiocyanate, and caffeine. J Natl Cancer Inst 1975;55:633-636.

[43] Frisch MJ, Trucks GW, Schlegel HB, et al. Gaussian 09 (C.01) inc. Wallingford CT 2009;

[44] Zheng YZ, Deng G, Liang Q, et al. Antioxidant activity of quercetin and its glucosides from propolis: A theoretical study. Sci Rep 2017;7:1-11.

[45] Muthukumaran J, Srinivasan S, Venkatesan RS, et al. Syringic acid, a novel natural phenolic acid, normalizes hyperglycemia with special reference to glycoprotein components in experimental diabetic rats. J Acute Dis 2013;2:304-309.

[46] Rimarčík J, Lukeš V, Klein E, et al. Study of the solvent effect on the enthalpies of homolytic and heterolytic $\mathrm{N}-\mathrm{H}$ bond cleavage in $\mathrm{p}$-phenylenediamine and tetracyano-p-phenylenediamine. $\mathrm{J}$ Mol Struct THEOCHEM 2010;952:25-30.

[47] Tomasi J, Mennucci B, Cammi R. Quantum mechanical continuum solvation models. Chem Rev 2005;105:2999-3093.

[48] Tomasi J, Mennucci B, Cance E. The IEF version of the PCM solvation method : an overview of a new method addressed to study molecular solutes at the QM ab initio level. 1999;464:211-226.

[49] Cramer CJ. Implicit Solvation Models: Equilibria, Structure, Spectra, and Dynamics - Chemical Reviews (ACS Publications). Chem Rev 1999;

[50] Kruszewski J, Krygowski TM. Definition of aromaticity basing on the harmonic oscillator model. Tetrahedron Lett 1972;13:3839-3842.

[51] Lee C, Yang W, Parr RG. Local softness and chemical reactivity in the molecules CO, SCN- and 
H2CO. J Mol Struct THEOCHEM 1988;163:305-313.

[52] Martinez C, Rivera JL, Herrera R, et al. Evaluation of the chemical reactivity in lignin precursors using the Fukui function. J Mol Model 2008;14:77-81.

[53] Ma Y, Liang J, Zhao D, et al. Condensed Fukui function predicts innate C-H radical functionalization sites on multi-nitrogen containing fused arenes. RSC Adv 2014;4:1726217264.

[54] Hanson RM. Jmol-a paradigm shift in crystallographic visualization. J Appl Crystallogr 2010;43:1250-1260.

[55] Humphrey W, Dalke A, Schulten K. VMD: Visual Molecular Dynamics. J Mol Graph 1996;14:33-38.

[56] Lu T, Chen F. Multiwfn: A multifunctional wavefunction analyzer. J Comput Chem 2012;33:580-592.

[57] Glendening ED, Landis CR, Weinhold F. NBO 6.0: Natural bond orbital analysis program. J Comput Chem 2013;34:1429-1437.

[58] Siquet C, Paiva-Martins F, Lima JLFC, et al. Antioxidant profile of dihydroxy- and trihydroxyphenolic acids - A structure-activity relationship study. Free Radic Res 2006;40:433442.

[59] Gordon MS, Jensen JH. Understanding the Hydrogen Bond Using Quantum Chemistry. Acc Chem Res 1996;29:536-543.

[60] Mazzone G, Toscano M, Russo N. Density functional predictions of antioxidant activity and UV spectral features of nasutin A, isonasutin, ellagic acid, and one of its possible derivatives. J Agric Food Chem 2013;61:9650-9657.

[61] Hamadouche S, Ounissi A, Baira K, et al. Theoretical evaluation of the antioxidant activity of some stilbenes using the Density Functional Theory. J Mol Struct 2021;1229:129496.

[62] Evgeny D, Taisa D. Dissociation Energies of O - H Bonds of. 2010;57:1858-1866.

[63] Alberti A, Amorati R, Campredon M, et al. Antioxidant activity of some simple phenols present in olive oil. Acta Aliment 2009;38:427-436.

[64] Mazzone G, Malaj N, Russo N, et al. Density functional study of the antioxidant activity of some recently synthesized resveratrol analogues. Food Chem 2013;141:2017-2024.

[65] Leopoldini M, Chiodo SG, Russo N, et al. Detailed investigation of the OH radical quenching by natural antioxidant caffeic acid studied by quantum mechanical models. J Chem Theory Comput 2011;7:4218-4233.

[66] Leopoldini M, Marino T, Russo N, et al. Antioxidant properties of phenolic compounds: H-atom versus electron transfer mechanism. J Phys Chem A 2004;108:4916-4922.

[67] Lin L, Zhu J. Antiaromaticity-Promoted Radical Stability in $\alpha$ - Methyl Heterocyclics. 2021;211.

[68] Ma Y, Feng Y, Diao T, et al. Experimental and theoretical study on antioxidant activity of the four anthocyanins. J Mol Struct 2020;1204:.

[69] Wright JS, Johnson ER, DiLabio GA. Predicting the activity of phenolic antioxidants: 
Theoretical method, analysis of substituent effects, and application to major families of antioxidants. J Am Chem Soc 2001;123:1173-1183.

[70] Fifen JJ, Nsangou M, Dhaouadi Z, et al. Solvent effects on the antioxidant activity of 3,4dihydroxyphenylpyruvic acid: DFT and TD-DFT studies. Comput Theor Chem 2011;966:232243.

[71] Chen J, Yang J, Ma L, et al. Structure-antioxidant activity relationship of methoxy, phenolic hydroxyl, and carboxylic acid groups of phenolic acids. 2020;1-9.

[72] Wang G, Xue Y, An L, et al. Theoretical study on the structural and antioxidant properties of some recently synthesised 2,4,5-trimethoxy chalcones. Food Chem 2015;171:89-97.

[73] Xue Y, Zheng Y, An L, et al. Density functional theory study of the structure-antioxidant activity of polyphenolic deoxybenzoins. Food Chem 2014;151:198-206.

[74] Urbaniak A, Szelag M, Molski M. Theoretical investigation of stereochemistry and solvent influence on antioxidant activity of ferulic acid. Comput Theor Chem 2013;1012:33-40.

[75] Ali A, Khalid M, Abid S, et al. Green synthesis, SC-XRD, non-covalent interactive potential and electronic communication via DFT exploration of pyridine-based hydrazone. Crystals 2020;10:120. 